\title{
HVAC-DYNAMIC-A training simulator for dynamic analysis of HVAC plants
}

\author{
MORTEN HEINTZ $\dagger$, VOJISLAV NOVAKOVIC $\dagger$, \\ OMMUND ØGARD $\ddagger$ and GEORG BRUSTAD
}

\begin{abstract}
Keywords: Air conditioning, modeling, dynamic analysis, software tool.
HVAC-DYNAMIC is a software tool for the dynamic simulation of Heating, Ventilation and Air Conditioning (HVAC) plants. The program is designed to be used by HVAC engineers during design or troubleshooting of plants and by plant operators in their training. The program is based on a set of the most-used HVAC plant configurations and requires only a minimum of knowledge in numeric methods and programming. A brief presentation of the program structure and examples showing some of the application of the program are given.
\end{abstract}

\section{Introduction}

Due to a combination of increased efforts on energy conservation and higher demands upon the indoor climate and air quality, research on dynamic behaviour of HVAC-systems and on improved control has intensified over the last few years. A general progress in data processing techniques and computerized control is also of great importance here.

In order to size components and to tune a control system to fit the plant layout, the dynamics of the plant and the control system have to be fairly well known. The best way to acquire such knowledge is through dynamic simulation of the actual plant. To make such simulations beneficial, the simulation tools should be given to the engineers engaged in the design work on the HVAC plants. This means that most effort during the design of the simulation program has to be made on the construction of a robust user interface, where as much of the numeric and computation details as possible are hidden from the user.

This paper presents briefly the program structure and the user interface of a dynamic simulation program called HVAC-DYNAMIC, together with an example of an application and some highlights of the model deduction and verification. HVACDYNAMIC is the successor of a simulation system used to test different controllers by physically connecting the controller to a simulated HVAC plant (on line emulation). More details about this work is given in Novakovic, Brustad and Øgärd 1986, Novakovic and Øgård 1986.

Received 1 August 1989.

†SINTEF Division of Applied Thermodynamic, HVAC Group, N-7034 Trondheim, Norway.

\$ SINTEF Division of Automatic Control, N-7034 Trondheim, Norway.

$\S$ Computer Aided Modelling, CAMO A/S, Jarlev. 4, N-7041 Trondheim, Norway.

This paper was presented at SIMS '89 (Scandinavian Simulation Society, 31st Annual Meeting, Bergen, Norway, May 31-June 2, 1989. 


\section{Simulation program}

\subsection{Program structure}

The major design criterion for the HVAC-DYNAMIC was to develop an easy to use simulation tool for testing the dynamic properties of HVAC plants both during the design and troubleshooting phases. Typical applications are tests of stability properties, controller performance and energy consumption with respect to plant structure, component sizes and controller tuning.

In order to make HVAC-DYNAMIC available to users without too much knowledge of programming and numeric methods, the program is based on a set of fixed plant configurations which includes the most commonly used HVAC plants. The user chooses the desired plant structure from a menu and is only allowed to modify the parameters of the components. Each plant configuration is equipped with some of the typical control structures and the user may choose the controller and tune the parameters. This approach is possible because the structures of most HVAC plant are standardized. It is assumed that about ten different configurations will be sufficient to cover the most plant layouts.

The real program structure is hidden behind the user menus and the final user is therefore not distracted by it. Different plant configurations are built up by connecting together component models taken from the model library into a plant configuration subroutine through a standard parameter passing interface. During this approach, the numeric solutions of the differential equations are simplified and optimized. The different configurations are programmed and solved separately using always the most appropriate method. This task, which requires some skills in FORTRAN programming and knowledge of the parameter passing interface, as well as a comprehensive knowledge of the internal behaviour or of numeric methods, is done by the program designer.

\subsection{User interface}

Man-machine interaction is menu-driven and is operated interactively by use of the cursor keys. The menu tree only has a few levels with a small number of commands on each level. The menu-driven interface is perfect for novices and users who are unfamiliar with programming and need guidance when operating the program. The instructions are only executed on request and will thus not lead to inefficient operation for an experienced user. Results are stored on file and may be presented graphically after the simulation. A selection of variables may be displayed while the simulation is running. HVAC-DYNAMIC has a separate configuration command where a sketch of the current plant is drawn on the screen and various control structures may be chosen.

Figure 1, shows the main menu for the program. Figure 2, shows the sketch of the control loop for the temperature control of a heat coil with a shunt valve. Figure 3, shows how the physical parameters of the duct model are represented in the program. The values may easily be changed by moving the cursor to the desired position and typing a new value. The other models are represented in the same way, and the user may turn from one model to the next by using the cursor keys. The asterisk '*' in the REP column indicates repetitive mode. In this mode HVAC-DYNAMIC automatically starts several simulations with different values of the marked parameter. The user specifies min and max values for the parameter and the number of repetitions. Figure 4, shows the outlet temperature for three different duct lengths. 


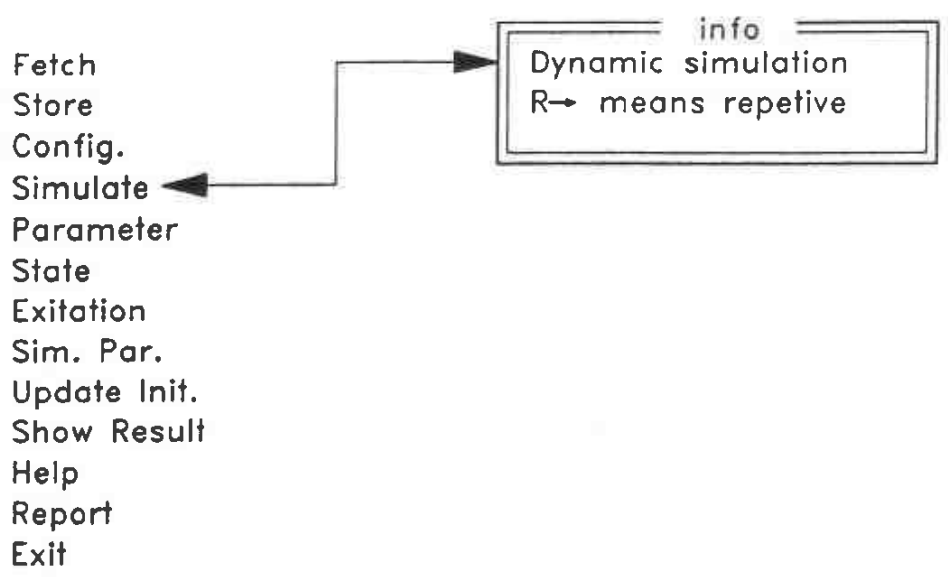

Figure 1. The main menu for the program.

CENTRAL SUBSYSTEM

Constant volume, Heating only, Secondary pump and three way valve

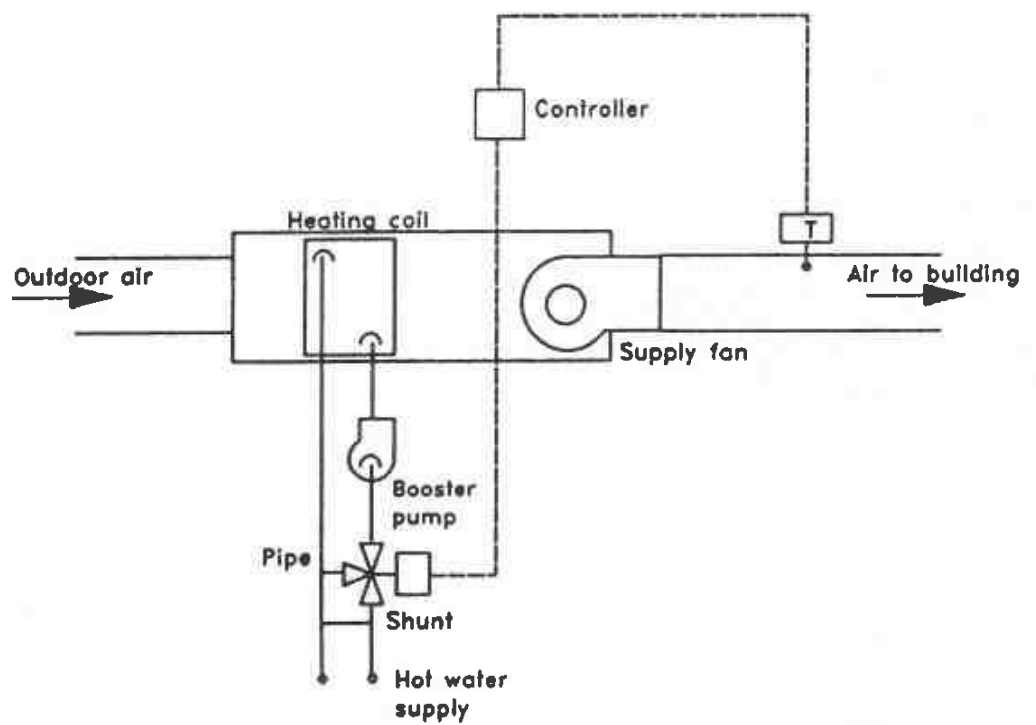

Figure 2. The sketch of the control loop for the temperature control of a heat coil with a shunt valve.

At every time step, states are tested against upper and lower limits which are specified by the plant constructor. By these means it is possible to terminate a simulation run properly and give error and warning measages. This applies, for instance, when the system is numerically unstable or physically unstable because of erroneous parameters.

HVAC-DYNAMIC próvides several types of input excitations, such as the outdoor climate, water supply temperature and occupancy pattern. Step changes, general functions and data stored on file may all be used. The general function may be approximations to typical meteorological data.

HVAC-DYNAMIC is written in standard FORTRAN and is available for IBMPC (MS-DOS) and compatible computers, and for VAX (VMS) machines. All facilities for user interaction, graphics and file processing are based on CYPROS-A Cybernetic Program System, developed and manufactured by CAMO A/S. 


\begin{tabular}{lrrl} 
Module: Duct1 & Volue & Rep. & Description \\
\hline Name & 7753 & & Duct density \\
ROM & 486 & & Spec. heat capasify of duct \\
0 & 0.511 & & Outside diameter \\
DR & 0.509 & & Inside diometer \\
H & 6.2 & & Heat transf. coef. air/wall \\
UAMB & 0 & & Heat transf. coef. wall/air \\
L & 10 & & Duct length \\
0 & 0.272 & & Flow in duct
\end{tabular}

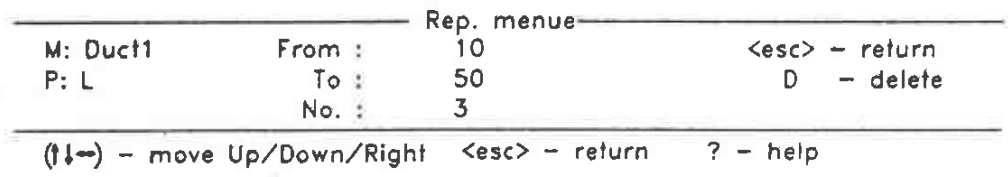

Figure 3. The menu for the physical parameters of the duct model.

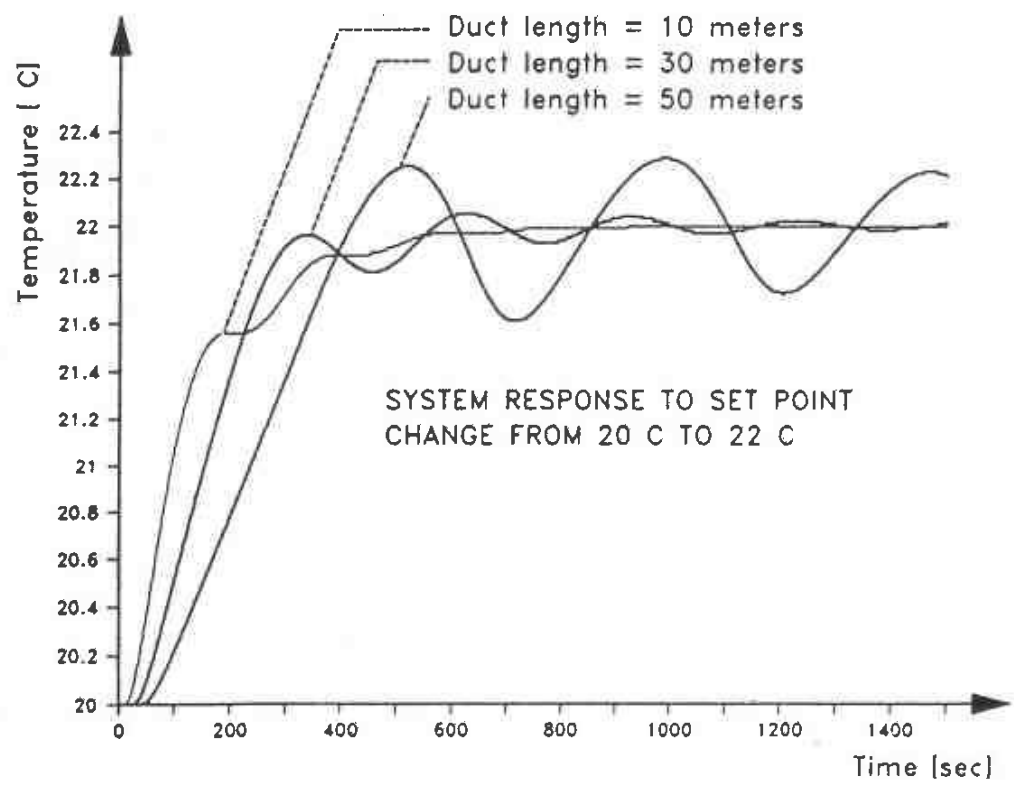

Figure 4. The outlet temperature for three different duct lengths.

\section{Component models}

\subsection{Model library}

During the development of HVAC-DYNAMIC great effort was concentrated on establishing the model library in order to produce efficient and accurate models with easily specified input parameters. The latter were obtained by making use of physical parameters such as pipe length, flowrate, heat capacity and so on instead of using gains, time delay and time constants. In order to reduce the number of parameters in each model, those which take a common value in several models are assembled within a separate module. Typical constant parameters are the heat capacities and densities of air and water which may be considered constant in the relevant temperature range. 
The model library today consists of the following: Pipe, Four port shunt coupling, Control valve, Heat coil, Duct, Fan, Heat recovery wheel, Mixing damper, Sensor, Actuator, Controller, Building and Environment.

So far, only plant configurations based on the four port shunt coupling are implemented. Due to the constant flow in such plants, most of the component models will be linear and first order apart from 'Building' which is second order. 'Pipe' and 'Duct' contain an additional time-delay between the input and output. The 'Shunt coupling' and the 'Control valve' are modeled as nonlinear functions without any dynamics. The 'Actuator' includes saturation and backlash, and may either be governed by a direct position reference or by an electric motor. More details about the models are given in Novakovic (1982) and Børresen and Thunem (1984).

\subsection{Model verification}

The models were verified by comparing measurements for a pilot plant and the corresponding results from the simulations. Measured input temperatures from the plant were used as input data to the numeric models in the simulations.

The layout of the pilot plant is quite similar to the plant shown in Fig. 2. This plant configuration is chosen because it is a commonly used layout which makes it possible both to verify the individual models one by one as well as the more compound parts of the plant. On the pilot plant it is possible to change the water temperature of the inlet supply into pipe and the air temperature of the inlet supply to the heat coil, by adjusting the set points of the local controllers for the boiler and for the air conditioning unit.

Four different series of measurements were performed using step changes in the temperatures of the supply water and of the inlet air and in the position of the shunt valve respectively. The fourth time series included perturbations of all the input variables by introducing step changes in a stochastic manner. The sampling frequency was $1 \mathrm{~Hz}$. All temperatures were measured using copper-constantan thermocouples and were logged by a $21 \mathrm{X}$ Micrologger from Campbell Scientific, Inc. The measurements were transformed to the CYPROS Time Series file format, also used by HVAC-DYNAMIC.

\section{Conclusion}

This paper has presented the simulation program HVAC-DYNAMIC and some bases for design of the various HVAC configurations. The models used are tested and verified against real world data from the pilot plant.

The primary goal was to develop a simulation tool for a wide range of users, from novices to experts, from plant operators to model constructors. The designed user interface is both easy to learn and yet flexible for the advanced user. The program is designed for use by HVAC engineers during design or trouble-shooting of plants and by plant operators in training purposes.

The idea of using a finite number of HVAC configurations simplifies users approach to the program considerably. The flexible program structure does not seriously restrict the development of new plant configurations which may easily be implemented by the program designer. 


\section{REFERENCES}

Børresen, B. A. and Thunem, AA. J. (1984). HVAC-control models for simulation SINTEF report STF48 A84019, Trondheim.

Novakovic, V. (1982). Digital Control of heating coils (in Norwegian). Dr. ing. Thesis, Norwegian Institute of Technology, Trondheim.

Novakovic, V., Brustad, H., and ØGARD, O. (1986). Adaptive controller for HVAC plant (in Norwegian). SINTEF report STF15 A86001, Trondheim.

NovaKovic, V. and ØGÅRD, O. (1986). HVAC-DYNAMIC-Program system for simulation and emulation. The Second International Conference on System Simulation in Buildings, December 1-3, 1986. Liege, Belgium, 221-232. 